\title{
Analysis, Fabrication, and Characterization of Tunable DFB Lasers with Chirped Gratings
}

\author{
Nong Chen, Member, IEEE, Yoshiaki Nakano, Member, IEEE, Kazuya Okamoto, Kunio Tada, Fellow, IEEE, \\ Geert I. Morthier, Member, IEEE, and Roel G. Baets, Senior Member, IEEE
}

\begin{abstract}
This paper describes the analysis, fabrication, and characterization of chirped grating tunable distributed-feedback (CGT DFB) semiconductor lasers. Both theoretical and experimental results show that the wavelength tunability of DFB lasers can be enhanced by introducing pitch-chirped grating structure. The tuning mechanism is also theoretically demonstrated. The proposed CGT DFB laser will be useful in optical systems using wavelength division multiplexed (WDM) networks and other applications, due to its broader continuous tunability and very easy fabrication.
\end{abstract}

Index Terms - Chirped grating, continuous tuning, DFB laser, linear chirp, tunable laser.

\section{INTRODUCTION}

A DISTRIBUTED-FEEDBACK (DFB) semiconductor laser with chirped grating (symmetric pitch chirp) has been theoretically proposed for decreasing threshold gain and improving single-mode selectivity by one of the authors before [1]. Nowadays, the chirped grating structures are also noted for their theoretically predicted superior abovethreshold characteristics compared with the $\lambda / 4$-phase-shifted grating structure. A linearly chirped grating (but symmetrically distributed in the cavity) DFB laser has been theoretically investigated by Zhou and Lee for mode selection and spatial hole burning suppression properties [2] or for narrow linewidth property in phase-shifted structure [3]. The same authors also analyzed the chirped grating (linear chirp in the whole cavity) $\lambda$ 4-shifted DFB laser for uniform longitudinal field distribution [4]. Pan et al., also analyzed the linewidth reduction of the $\lambda / 4$-shifted DFB laser with chirped gratings and demonstrated a linewidth reduced effect introduced by symmetrically chirped grating and a linewidth increased effect caused by asymmetrically chirped grating [5]. In addition, the theoretical analyses of the chirped grating filter [6] and distributed Bragg reflection (DBR) structure [7] have also been reported.

Manuscript received November 26, 1996; revised March 27, 1997. This work was supported in part by a Kurata Research Grant and in part by Mombusho Grant-in-Aid 08044123.

N. Chen is with the Devices and Materials Department, Corporate Research and Development Laboratory, Pioneer Electronic Corporation, Saitama 35002, Japan.

Y. Nakano and K. Tada are with the Department of Electronic Engineering, University of Tokyo, Tokyo 113, Japan.

K. Okamoto is with the Research and Development Department, Nikon Corporation, Tokyo 140, Japan.

G. I. Morthier and R. G. Baets are with INTEC, University of Gent, Gent B 9000, Belgium.

Publisher Item Identifier S 1077-260X(97)04594-2.
In experiments, the initial properties of an optically pumped laser emission were reported ten years ago [1]. The lasing demonstration of the chirped grating DFB laser formed by a bent waveguide structure has also been reported recently [8]. A DFB laser with corrugation-pitch-modulated (CPM) structure has been shown, both theoretically and experimentally, with superior spectral linewidth properties [9]. For wavelength tunable optical devices, the chirped grating has been proposed as a tunable filter in theory [10] and has also been applied in a super structure grating (SSG) [11] sampled DBR laser.

DFB lasers cannot achieve as good tuning as DBR devices, but DFB types do not give a large linewidth or rather FM noise and probably also give high speed. Additionally, the DFB type has the merit of simple fabrication. We have proposed to use chirped grating for extending the wavelength tuning range of DFB lasers and have reported some results about the lasing properties and tuning properties of chirped grating DFB lasers [12]-[14]. In this paper, to demonstrate the enhanced wavelength tunability of the chirped grating tunable (CGT) DFB laser, the theoretical analyses of wavelength tuning properties are first described. The fabrication and characterization of CGT DFB lasers with different chirps are then reported.

\section{THEORETICAL ANALYSIS}

The tunable lasers with passive tuning region like tunable DBR lasers, tunable twin-guide (TTG) lasers, and other types including phase-tunable DFB lasers, have relatively simple tuning mechanisms and have been fully theoretically analyzed [15]-[17]. Some papers also theoretically discussed the properties of multielectrode DFB lasers [18]-[22]. In this paper, with the aide of CLADISS (Compound LAser DIode Simulation Software) [23], the tuning mechanism and the tuning properties of CGT DFB laser are discussed and numerically demonstrated. The simulated wavelength tuning of a conventional uniform grating DFB laser for comparison agrees well with the reported experimental result for the first time, also.

In CGT DFB lasers, the wavelength tuning is governed not only by the refractive index change, which has been the tuning mechanism of most conventional tunable DFB lasers, but also by the chirped grating effect; when the photons are localized in the cavity, they feel a pitch of the chirped grating at that particular position. As the position of the localization changes by any means, the effective pitch changes accordingly. The photon density distribution profile can be modified by changing the current injection ratio between different sections 


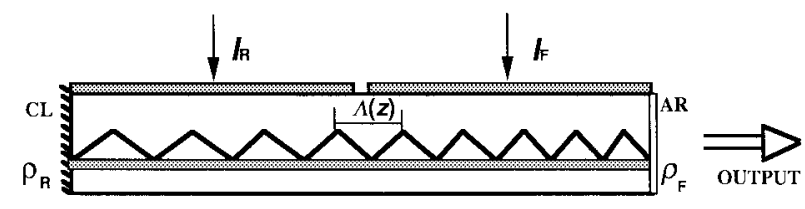

Grating Pitch: $\quad \Lambda(z)=\Lambda(0)\left(1+D \frac{z}{L}\right)$
Chirp Parameter: $D=\frac{\Lambda(L)-\Lambda(0)}{\Lambda(0)}$

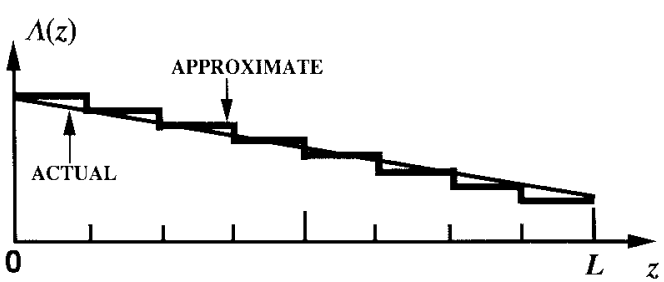

Fig. 1. Schematic drawing of the two-electrode CGT DFB laser for numerical calculations.

of the multielectrode DFB laser. This causes an additional wavelength shift introduced by the chirped grating, except for the index variation that can be induced by both carrier density change and photon density change in DFB lasers.

A simple case of a two-section DFB laser with the rear facet as cleaved (CL) and the front facet as antireflection (AR) is considered here. Its linearly chirped grating is approximated by a stepped chirped grating, as shown in Fig. 1, in the calculations. The coupled mode equations are used together with the carrier density rate equation of each longitudinal mode [23], by taking into account the nonuniformity of carrier density distribution along the cavity axis $z$ caused by nonuniform current injection and other effects like spatial hole burning. Most parameters used in the calculations are listed in Table I. The parameters are not optimized, and the influences of grating phase and confinement factor are not involved in this paper. In a linear system, the choice of confinement factor is not critical to the analysis of wavelength tuning. A large value of confinement factor has been used to allow in an approximate way for the effect of carriers in the adjacent waveguiding layer.

The simulated wavelength tuning characteristics for 1.55$\mu \mathrm{m}$ band lasers are shown in Fig. 2. The output power is kept as a constant of $1 \mathrm{~mW}$ when the injection current ratio is changed. The heat effect is not considered here, because a good heat sink is used in the experiment. A calculated tuning range of $2.7 \mathrm{~nm}$ (the dotted curve in the figure) for the conventional uniform grating DFB laser agrees well with the experimental value reported [24]. The plotted curve with filled squares shows the case of a CGT DFB laser with the linearly chirped grating, in which the grating pitch becomes gradually small from the rear side to the front side, and the chirp parameter or chirp rate (as defined in the Fig. 1) is $D=-1.66 \times 10^{-3}$. The chirped grating effect in this case is to tune the wavelength in a blue-shift direction as the same to that of the index effect when the injection current ratio of $I_{F} /\left(I_{F}+I_{R}\right)$ is increased. Therefore, the tuning range is broader than that of the uniform grating case. For the reverse case of a linearly chirped grating with $D=+1.66 \times 10^{-3}$
TABLE I

PaRAmeters Used in CALCUlations

\begin{tabular}{lll}
\hline \multicolumn{1}{c}{ Parameters } & Symbles & Values \\
\hline Laser stripe width & $w$ & $1.5 \mu \mathrm{m}$ \\
Active layer thickness & $d$ & $0.12 \mu \mathrm{m}$ \\
Laser cavity length & $L$ & $400 \mu \mathrm{m}, \ldots$ \\
Bragg order & $m$ & $1, \ldots$ \\
Grating period & $\Lambda_{o}$ & $0.2413 \mu \mathrm{m}$ \\
Field amplitude reflectivity at rear facet & $\rho_{R}$ & $0.565, \ldots$ \\
Field amplitude reflectivity at front facet & $\rho_{F}$ & $0, \ldots$ \\
Phase of reflectivity at rear facet & $\phi_{R}$ & $0, \ldots$ \\
Phase of reflectivity at front facet & $\phi_{F}$ & $0, \ldots$ \\
Coupling coefficient - Backward to & $\kappa_{F B}$ & $50 \mathrm{~cm}^{-1}$ \\
$\quad$ Forward propagating wave & & \\
Coupling coefficient - Forward to & $\kappa_{B F}$ & $50 \mathrm{~cm}^{-1}$ \\
$\quad$ Backward propagating wave & & 3.25 \\
Unperturbated effective refractive index & $n_{o}$ & $50 \mathrm{~cm}^{-1}$ \\
Internal waveguide losses coefficient & $\alpha_{l o s s}$ & 0.5 \\
Power confinement factor in active layer & $\Gamma$ & 0.8 \\
Current injection efficiency & $\eta$ & $5 \times 10^{-9} \mathrm{~s}$ \\
Carrier lifetime & $\tau$ & $1.6 \times 10^{-19}$ \\
Electron charge & $e$ & 100 \\
Bimolecular recombination rate & $B$ & $20 \times 10^{-5}$ \\
Auger recombination coefficient & $C$ & \\
\hline & & \\
\hline
\end{tabular}

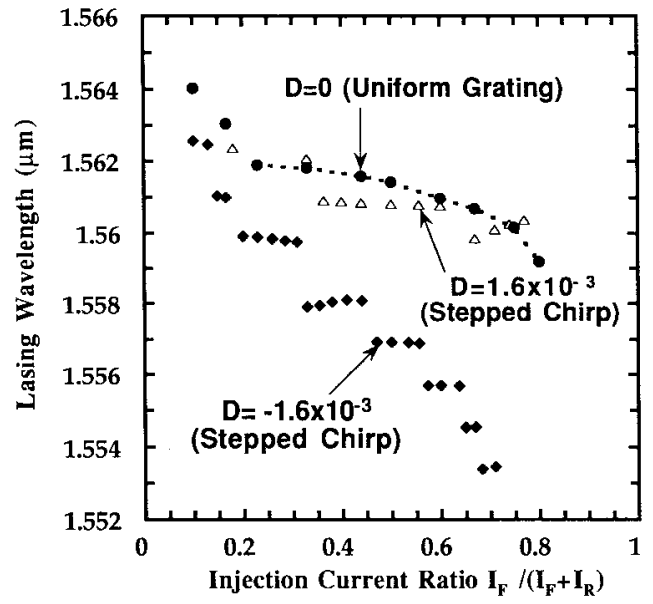

Fig. 2. Simulated tuning characteristics of the two-electrode DFB lasers with different gratings at $1-\mathrm{mW}$ output.

(plotted with triangles in the figure), the wavelength tuning effect of the chirped grating is opposite to that of the index effect, and the resulting tuning range is narrower than that of the uniform grating DFB laser. It is understood that the chirp rate $D$ should be chosen appropriately, in order to assure a 


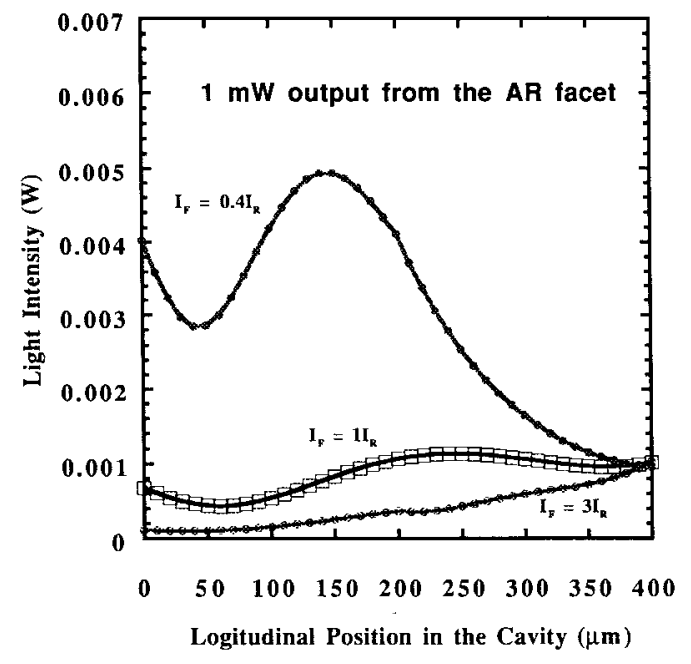

(a)

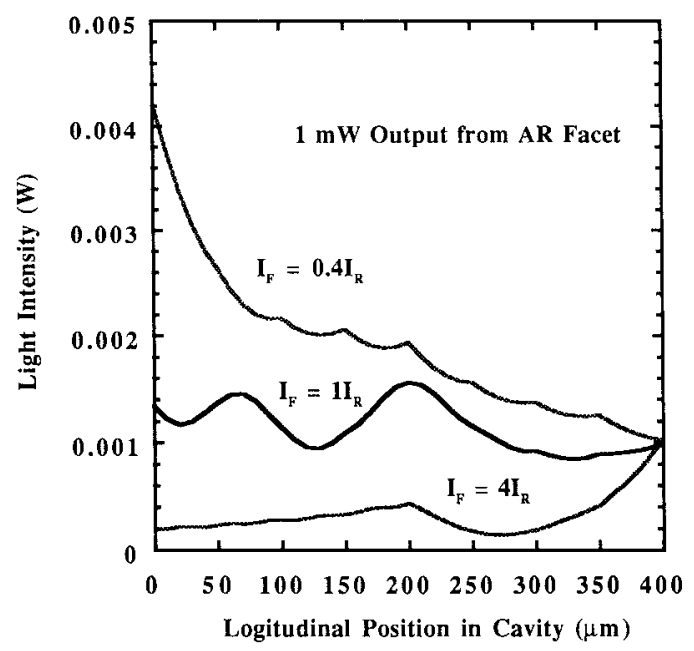

(b)

Fig. 3. Calculated photon density profiles at different bias conditions in the two-electrode DFB lasers. (a) With uniform grating. (b) With linearly chirped grating.

large tuning range. Negative $D$ is apparently preferable in this particular example.

For demonstration of the tuning mechanisms, the computed photon density profiles at different bias conditions are illustrated in Fig. 3(a) for the case of a uniform grating DFB laser and in Fig. 3(b) for the linearly chirped grating case. It is known that the photon localization is controllable through the ratio of injection current into different sections. This may cause a wavelength shift, in addition to the conventional index effect mentioned above, if the grating pitch is gradually changed along the cavity, while, in the uniform grating DFB lasers, the effective local Bragg frequency is only changed by the refractive index effect caused by the current tuning and spatial hole burning. This results in wavelength tuning by index change. Some calculated results of the refractive index distributions, under different bias conditions, along the two-electrode uniform grating DFB laser cavity are shown in Fig. 4. The output power is also kept as a constant of $1 \mathrm{~mW}$. It is known that the total refractive index decreases as the abovementioned injection current ratio increases. This will tune the lasing wavelength of the DFB laser in a blue-shift direction.

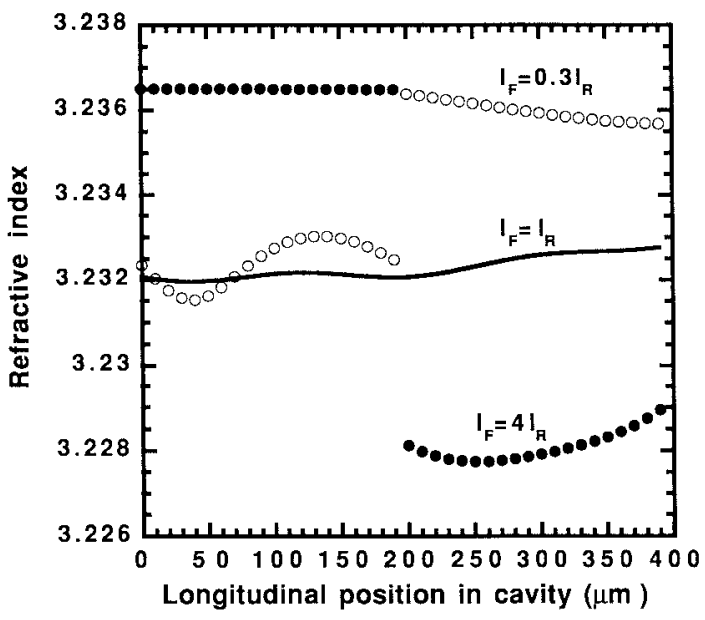

Fig. 4. Calculated refractive index distributions in two-electrode uniform grating DFB laser.

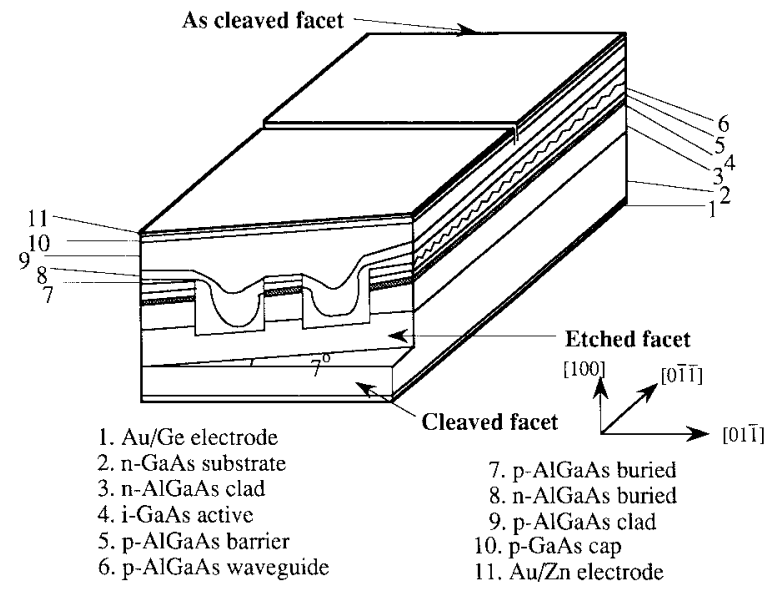

Fig. 5. Structure of the fabricated two-electrode CGT DFB laser.

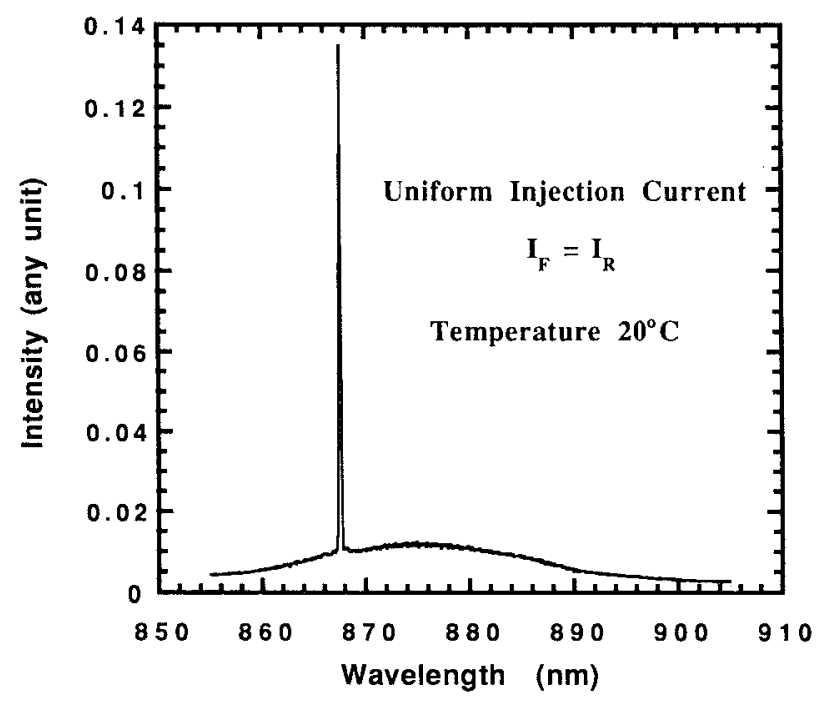

Fig. 6. Typical spectrum of a CGT DFB laser.

\section{EXPERIMENTAL RESULTS}

A simple two-electrode AlGaAs-GaAs CGT DFB laser with a bulk active layer, as shown in Fig. 5, has been fabricated for verifying the enhanced tuning effect introduced by the chirped grating structure, as discussed above. The spherical 


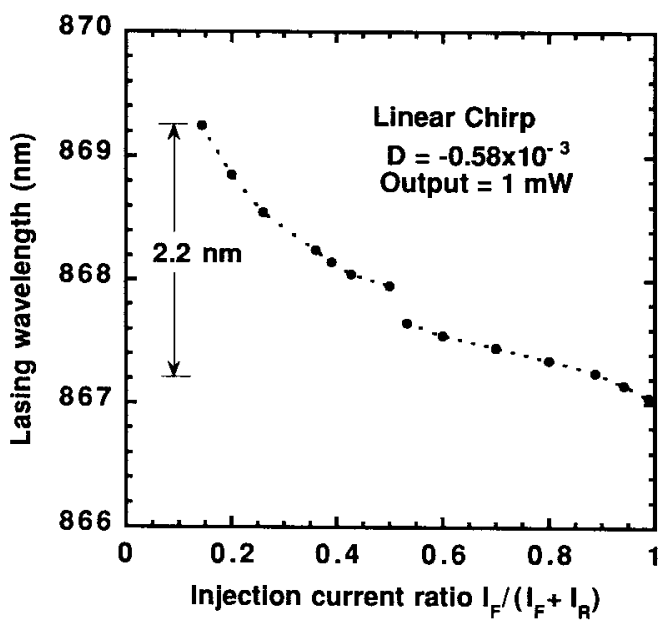

(a)

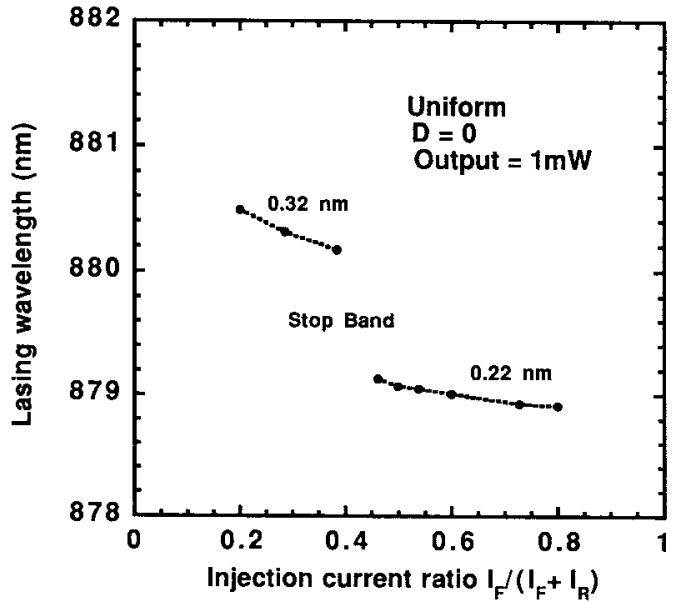

(b)

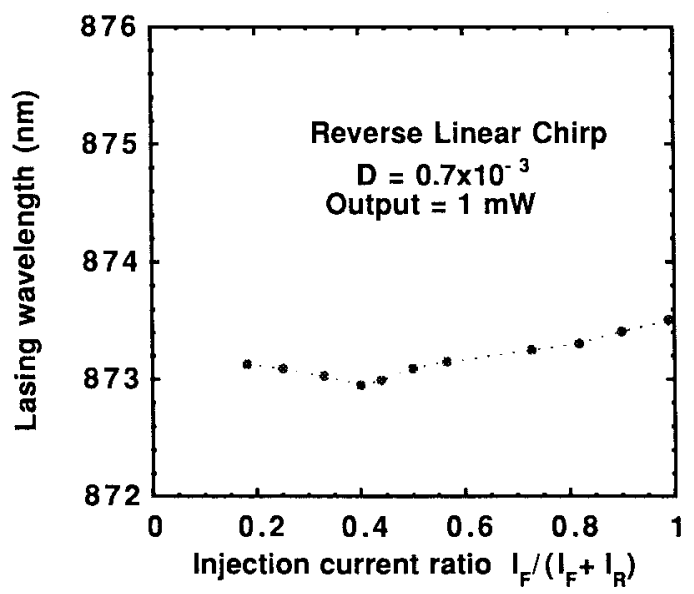

(c)

Fig. 7. Experimental tuning characteristics of the two-electrode GaAs DFB lasers with different gratings. (a) Linear chirp (negative $D$ ). (b) Uniform $(D=0)$. (c) Reverse linear chirp (positive $D)$.

wave holographic exposure method [25] has been applied for fabricating a second-order (with central pitch of around $0.255 \mu \mathrm{m})$ linearly chirped grating in the waveguide layer. With this method, the chirp rate of the grating pitch can be chosen easily. A proper photoresist is useful in the formation of high-contrast periodic corrugations [26]. Two-step liquid phase epitaxy (LPE) growth has been used for forming a doublechannel planar heterostructure (DCPBH). The front laser facet was etched with reactive ion etching (RIE) to form a $7^{\circ}$-angled mirror for reducing the reflectivity, instead of AR coating [27]. The rear facet was cleaved to form a cavity structure, as described in the calculating model.

One typical spectrum of the fabricated CGT DFB laser under uniform current injection is shown in Fig. 6. It corresponds to the chirped grating structure with an obvious Bragg wavelength detuning from the gain peak. In the spherical wave holographic exposure method for linearly chirped grating fabrication, the different position of the wafer sample has a different pitch. Usually, the grating pitch at the central position of the wafer is designed to match the laser gain peak in our experiments.

For comparison of the tuning properties, three kinds of fabricated two-electrode GaAs DFB lasers with linear chirp (negative $D$ ), uniform $(D=0)$, and reverse linear chirp (positive $D$ ) gratings have been characterized. The typical tuning results are shown in Fig. 7. Besides the grating, they have identical structures (including AR-CL facets) and fabrication conditions. All measurements were conducted with pulsed injection currents and with a good heat sink, so that thermal tuning could be neglected. Also, the total current was kept nearly constant, i.e., the output power was kept around $1 \mathrm{~mW}$ when the tuning characteristics were plotted. The neglectable thermal tuning effect is proved by the very small tuning shown in the uniform grating sample.

The index tuning effect in GaAs lasers is small compared to InP lasers, since the free carrier plasma effect is small. This is also proved by the very narrow continuous tuning range of the uniform grating device in Fig. 7(b). Therefore, the wavelength tuning in CGT DFB lasers could mainly be due to the chirped grating effect. An appropriate linear chirping in Fig. 7(a) gives rise to an extra tuning that amounts to approximately $2 \mathrm{~nm}$ at the $870-\mathrm{nm}$ band. This may correspond to about $4 \mathrm{~nm}$ in longer wavelength lasers of $1.55-\mu \mathrm{m}$ band and is very broad as quasi-continuous wavelength tuning for bulk two-electrode DFB lasers.

On the other hand, the reverse linear chirp in Fig. 7(c) brings about a net wavelength shift toward the opposite direction, 
after canceling the shift due to the index change. This is consistent with the theoretical results shown in Fig. 2.

Further work is needed to optimize the grating structure, like the magnitude of the grating chirp or phase-shift, so as not to introduce modal instability.

\section{CONCLUSION}

The wavelength tuning simulations of both CGT DFB lasers and uniform grating tunable DFB lasers have been demonstrated. It is known that the linearly chirped grating DFB laser with nonsymmetric cavity structure can bring about a broader wavelength tuning range with a proper choice of chirp parameter. The extended wavelength tuning of our proposed CGT DFB laser has been explained by the calculations of photon density distributions under different current injection ratios. It is also the first time that a simulated result of the tunable DFB laser with conventional uniform grating has been in very good agreement with the experimental tuning range reported. In experiments, AlGaAs-GaAs CGT DFB lasers with different linear chirps have been fabricated. A tuning range of $2.2 \mathrm{~nm}$ in a two-electrode CGT DFB laser has been observed by injection current tuning, which is equivalent to about a 4-nm tuning range in a $1.55-\mu \mathrm{m}$ wavelength band. This relatively large quasi-continuous tuning (with one very small mode hopping) is mainly considered as the effect of the linearly chirped grating structure. The extended tunability of the CGT DFB laser has also been confirmed by comparing it with the conventional uniform grating DFB laser fabricated under the same conditions and reconfirmed by the CGT DFB laser with reverse linear chirp. The CGT DFB lasers may be useful in future WDM systems and other applications. The chirped grating structure may also be helpful in enhancing the tunability of other tunable lasers like the DBR type, and so on.

\section{REFERENCES}

[1] A. Suzuki and K. Tada, "Theory and experiment on distributed feedback lasers with chirped grating," Proc. SPIE, vol. 239, pp. 10-18, 1980.

[2] P. Zhou and G. S. Lee, "Mode selection and spatial hole burning suppression of a chirped grating distributed feedback laser," Appl. Phys. Lett., vol. 56, no. 15, pp. 1400-1402, Apr. 1990.

[3] _ , "Phase-shifted distributed feedback laser with linearly chirped grating for narrow linewidth and high-power operation," Appl. Phys. Lett., vol. 58, no. 4, pp. 331-333, Jan. 1991.

[4] _ "Chirped grating $\lambda / 4$-shifted distributed feedback laser with uniform longitudinal field distribution," Electron. Lett., vol. 26, no. 20, pp. 1660-1661, Sept. 1990.

[5] X. Pan, H. Olesen, and B. Tromborg, "Linewidth reduction of $\lambda / 4$ shifted DFB lasers by the use of chirped gratings," in Tech. Dig. Conf. Lasers Electro-Opt. (CLEOO91), CWF15, Baltimore, MD, May 1991, pp. 252-253.

[6] H. Kogelnik, "Filter response of nonuniform almost-periodic structures," Bell Syst. Tech. J., vol. 55, pp. 109-126, Jan. 1976.

[7] Y. Nagashima, T. Sato, and N. Yamauchi, "Analysis of active DBR structure with chirped grating," Trans. Inst. Electron. Inf. Commun. Eng. C, vol. J75-C-I, no. 11, pp. 730-732, Nov. 1992.

[8] H. Hillmer, K. Magari, and Y. Suzuki, "Chirped grating for DFB laser diodes using bent waveguides," IEEE Photon. Technol. Lett., vol. 5, pp. 10-12, Jan. 1993

[9] M. Okai, M. Suzuki, and T. Taniwatari, "A corrugation-pitch-modulated strained multiple-quantum-well distributed feedback laser with an ultra narrow (3.6 kHz) spectral linewidth," in Proc. Extended Abstracts 1993 Int. Conf. Solid State Dev. Mat. (SSDM'93), Makuhari, Japan, Sept. 1993, pp. 44-46.

[10] T. Schrans, M. Mittelstein, and A. Yariv, "Tunable active chirpedcorrugation waveguide filters," Appl. Phys. Lett., vol. 55, no. 3, pp.
212-214, July 1989

[11] H. Ishii, Y. Tohmori, T. Tamamura, and Y. Yoshikuni, "Super structure grating (SSG) for broadly tunable DBR lasers," IEEE Photon. Technol. Lett., vol. 4, pp. 393-395, Apr. 1993.

[12] N. Chen, Y. Nakano, and K. Tada, "Chirped grating tunable (CGT) DFB laser-Novel laser diode with broader tuning range," in Tech. Dig. SPIE Int. Conf. Lasers and Opto-Elec. (ICLOEO92), Beijing, China, Oct. 1992, vol. 2A10, pp. 73-73.

[13] _ "Fabrication of multiple-electrode chirped-grating-tunable distributed-feedback lasers," Jpn. J. Appl. Phys., Regul. Rap. Short Notes vol. 33, no. 1B, pp. 856- 858, Jan. 1994.

[14] N. Chen, Y. Nakano, K. Tada, K. Okamoto, G. Morthier, and R. Baets, "Enhanced tunability in chirped grating DFB lasers," in Proc. IEEE LEOS'94 Conf., 1994, vol. 1, pp. 101-102.

[15] X. Pan, H. Olesen, and B. Tromborg, "A theoretical model of multielectrode DBR lasers," IEEE J. Quantum Electron., vol. 24, pp. 2423-2432, Dec. 1988.

[16] M.-C. Amann, S. Illek, C. Schanen, and W. Thulke, "Tuning range and threshold current of the tunable twin-guide (TTG) laser," IEEE Photon. Technol. Lett., vol. 1, pp. 253-254, Sept. 1989.

[17] H. Olesen, X. Pan, and B. Tromborg, "Theoretical analysis of tuning properties for a phase-tunable DFB laser," IEEE J. Quantum Electron., vol. 24, pp. 2367-2375, Dec. 1988.

[18] M. Kuzentsov, "Theory of wavelength tuning in two-segment distributed feedback lasers," IEEE J. Quantum Electron., vol. 24, pp. 1837-1844, Sept. 1988.

[19] K. Kikuchi and H. Tomofuji, "Performance analysis of separatedelectrode DFB laser diode," Electron. Lett., vol. 25, no. 2, pp. 162-163, Jan. 1989.

[20] _ "Analysis of oscillation characteristics of separated-electrode DFB laser diodes," IEEE J. Quantum Electron., vol. 26, pp. 1717-1727, Oct. 1990.

[21] H. Shoji, Y. Arakawa, and Y. Fujii, "Theoretical analysis of twoelectrode DFB lasers considering spatial hole burning and gain saturation," in Tech. Dig. 3rd Optoelec. Conf. (OEC'90), Makuhari, Japan, July 1990, vol. 12B2-1, pp. 74-75.

[22] M. Tohyama, M. Onomura, M. Funemizu, and N. Suzuki, "Wavelength tuning mechanism in three-electrode DFB lasers," IEEE Photon. Technol. Lett., vol. 5, pp. 616-618, June 1993.

[23] P. Vankwikelberge, G. Morthier, and R. Baets, "CLADISS-A longitudinal multimode model for the analysis of the static, dynamic, and stochastic behavior of diode lasers with distributed feedback," IEEE J. Quantum Electron., vol. 26, pp. 1728-1741, Oct. 1990.

[24] M. Okai, S. Sakano, and N. Chinone, "Wide-range continuous tunable double-sectioned distributed feedback lasers," in Proc. 15th Euro. Conf. Opt. Comm. (ECOCO89), Gothenburg, Sweden, Jan. 1989, pp. 122-125.

[25] A. Suzuki and K. Tada, "Fabrication of chirped gratings on GaAs optical waveguides," Thin Solid Films, vol. 72, pp. 419-426, 1980

[26] K. Okamoto, Y. Nakano, and K. Tada, "Formation of high-contrast periodic corrugations by optimizing optical parameters of photoresists in $325 \mathrm{~nm}$ laser holographic exposure," Jpn. J. Appl. Phys., vol. 34, no. 2B, pp. 1286-1292, Feb. 1995.

[27] Y. Nakano, Y. Hayashi, N. Chen, Y. Sakaguchi, and K. Tada, "Fabrication and characteristics of an integrated DFB laser/amplifier having reactive-ion-etched titled end facets," Jpn. J. Appl. Phys., vol. 29, no. 12, pp. L2430-L2433, Dec. 1990.

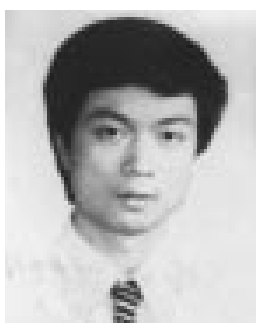

Nong Chen (S'90-M'93), was born in China in 1962. He received the B.S. and M.S. degrees in physics from the University of Science and Technology of China (USTC), Hefei, China, in 1985 and 1988, respectively, and the Ph.D. degree in electronic engineering from the University of Tokyo, Tokyo, Japan, in 1994

In 1994, he joined the Corporate Research and Development Laboratory, Pioneer Electronic Corporation, Saitama, Japan, where he is currently engaged in the research and development of new laser diode devices. His research interests include holography, distributed feedback semiconductor lasers, and integrated devices. He has authored or coauthored 40 papers and conference presentations.

Dr. Chen is a member of the Japan Society of Applied Physics. He was the recipient of the 1993 Kodi Husimi Prize from the Japan-China Science and Technology Association. 


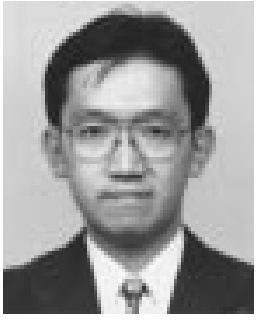

Yoshiaki Nakano (S'81-M'87) was born in Tokyo, Japan, on May 4, 1959. He received the B.E., M.S., and Ph.D. degrees in electronic engineering, all from the University of Tokyo, Japan, in 1982, 1984, and 1987, respectively. In 1984, he spent a year at the University of California, Berkeley, as an exchange student.

In 1987, he joined the Faculty of Engineering, the University of Tokyo, and is currently an Associate Professor with the Department of Electronic Engineering. His research interests have been physics and fabrication technologies of semiconductor distributed-feedback lasers and photonic integrated circuits. In 1992, he was a visiting Associate Professor at the University of California, Santa Barbara.

Dr. Nakano is a member of the Institute of Electronics, Information, and Communication Engineers, the Japan Society of Applied Physics, and the Japan Institute for Interconnecting and Packaging Electronic Circuits. He is the recipient of the 1987 Shinohara Memorial Prize from the IEICE and the 1991 Optics Paper Award from the JSAP.

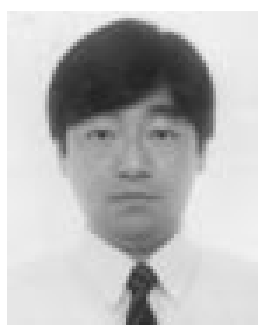

Kazuya Okamoto received the Ph.D. degree in electronic engineering from the University of Tokyo, Tokyo, Japan, in 1996.

$\mathrm{He}$ is currently the Manager of the Microdevice Research Group, Nikon Corporation, Tokyo, Japan. His research interests include design, fabrication, and characteristic analysis in waveguide devices applicable to optical measurement systems.

Dr. Okamoto is a member of the Japan Society of Applied Physics.

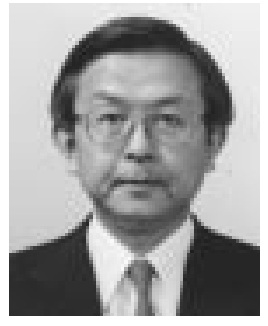

Kunio Tada (S'60-M'65-SM'84-F'89) was born in Tokyo, Japan, on March 31, 1938. He received the B.E. degree in electrical engineering, and the M.E. and $\mathrm{Ph} . \mathrm{D}$. degrees in electronic engineering from the University of Tokyo, Tokyo, Japan, in 1960, 1962 , and 1965 , respectively.

He joined the Faculty of Engineering, University of Tokyo in 1965 as Lecturer. In 1966, he became Associate Professor of the Department of Electronic Engineering, and since 1981, he has been serving as Professor. From 1972 to 1973, he was with the University of Pennsylvania, Philadelphia, PA. He had conducted research on semiconductor devices such as high-speed bipolar transistors, Schottkyclamped transistors, and integrated optics. In 1966, he began research on electrooptic materials and modulators. Presently, he is directing a research group working on distributed-feedback lasers including those with gain coupling, semiconductor integrated-optic modulators/switches, semiconductor photonic integrated circuits, etc.

Dr. Tada is serving as President of the Japan Society of Applied Physics, and is a member of the Engineering Academy of Japan, Physical, Society of Japan, the Institute of Electronics, Information, and Communication Engineers, the Institute of Electrical Engineers of Japan, the Japan Institute of Printed Circuit, the Japan Society of Engineering Education, and the Optical Society of America. He was awarded the Hattori-Houkou Prize in 1987 for his contribution to the first implementation of the optical directional-coupler modulator/switch published in 1974

Geert I. Morthier (M'93), photograph and biography not available at the time of publication.

Roel G. Baets (M'88-SM'96), photograph and biography not available at the time of publication. 